\title{
NOUVELLE
}

\section{Un héritage inégal \\ à la base des fonctions effectrice ou mémoire des descendants des lymphocytes T}

Nathalie Auphan-Anezin
Centre d'Immunologie de Marseille-Luminy (CIML), Aix-Marseille Université, Faculté des Sciences de Luminy, Inserm U631, CNRS UMR6102, Campus de Luminy, Case 906, 13288 Marseille Cedex 09, France. auphan@ciml.univ-mrs.fr
> L'interaction des lymphocytes T naïfs avec des cellules présentatrices de l'antigène (CPA), communément les cellules dendritiques, dans les organes lymphoïdes secondaires, induit leur activation. La microscopie confocale a permis de mettre en évidence la ségrégation des molécules d'activation à l'interface entre le lymphocyte $T$ et la CPA, une structure appelée «synapse immunologique » [1-3] (Figure 1).

Par ailleurs, depuis 2002, plusieurs groupes ont utilisé les techniques de microscopie multiphotonique afin de visualiser, à l'intérieur d'un ganglion lymphatique intact, les déplacements des lymphocytes $T$ et leur interaction avec les CPA en l'absence ou en présence de l'antigène (Ag) [4]. Les premières heures $(0-6 h)$ qui suivent l'entrée des lymphocytes dans un ganglion lymphatique sont caractérisées par des interactions de courte durée avec les CPA, qui induisent l'expression de marqueurs d'activation. La seconde phase (6-32h) implique une interaction de longue durée (> 1h) avec une CPA présentant l'Ag et l'initiation de la sécrétion de cytokines, en l'absence de division cellulaire. Dans la troisième phase, les lymphocytes $T$ se détachent des CPA, prolifèrent et retrouvent une grande mobilité (Figure 2).

L'activation des lymphocytes $T$ induit un programme développemental incluant à la fois une phase d'expansion clonale et la différenciation des cellules $T$ naïves en cellules $T$ effectrices ou mémoires. La généalogie des cellules mémoires est, encore à ce jour, sujet de controverses et 3 modèles sont proposés [5] :
- le premier propose une différenciation divergente des cellules effectrices et mémoires, qui serait influencée par la durée de la stimulation antigénique initiale [6] et les cytokines environnantes, notamment de type inflammatoire;

- le second est une différenciation linéaire des cellules T d'abord en cellules effectrices, puis de façon ultime en cellules mémoires;

- le troisième est une variation du modèle précédent, qui intègre en plus une relation inversement corrélée entre la durée de la stimulation antigénique et la capacité des cellules T à se différencier en cellules mémoires.

Jusqu'à présent, aucune étude n'avait fait le lien entre les évènements précoces qui surviennent au niveau de la synapse immunologique et les évènements tardifs qui régissent, après la phase d'expansion clonale, la différenciation en cellules T effectrices ou mémoires. Cependant, l'observation que les lymphocytes $T$ restent en contact pendant plusieurs heures avec la CPA suggérait que cette interaction englobe la phase de mitose. Dans un numéro récent de Science [7], I'équipe de Steven Reiner propose que les cellules $T$ effectrices et mémoires soient simultanément générées lors de la première mitose qui suit la reconnaissance de l'Ag selon un mécanisme de division asymétrique.

Des lymphocytes $T$ exprimant un TCR ( $T$ cell receptor) spécifique d'un Ag de Listeria monocytogenes ont été marqués avec un composant fluorescent et injectés à une souris infectée. Trente-deux heures

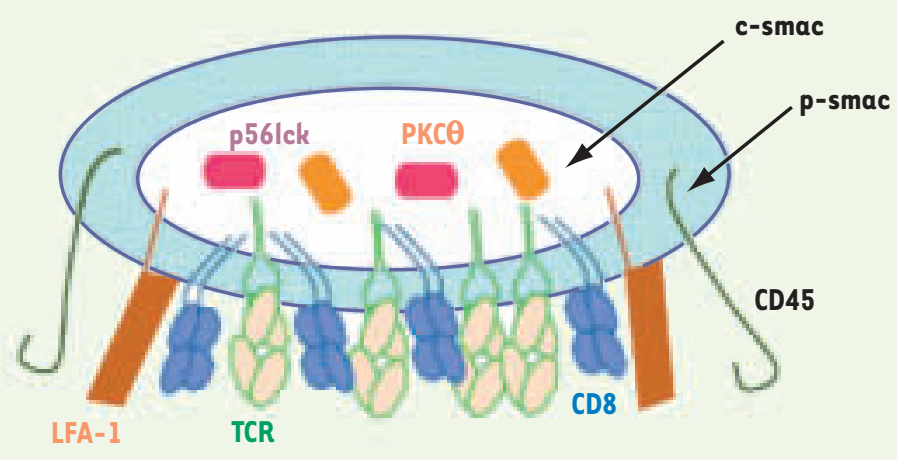

Figure 1. Synapse immunologique. La zone centrale de la synapse (c-smac) concentre notamment le récepteur à l'antigène (TCR) et les molécules CD3 associées, les co-récepteurs CD4 ou CD8, les protéines kinase PKC $\theta$ et $p 561 c k$; à la périphérie de cette zone centrale, se trouvent les molécules d'adhérence LFA-l et la taline; enfin, une zone distale ( $p$-smac) contient des molécules de grande taille telle la phosphatase CD45. Très tôt dans la formation de la synapse, il y a une polymérisation localisée du cytosquelette d'actine et le centre organisateur des microtubules (MTOC) se réoriente vers la synapse (non montré). 
après leur transfert, les lymphocytes $T$ qui, à ce stade, ne se sont pas encore divisés mais ont subi une augmentation de leur volume (phénotype de blastes) en réponse à leur activation, sont analysés en microscopie confocale. Dans la plupart de ces cellules T activées pré-mitotiques, le MTOC (microtubule-organizing center) co-localise avec les molécules LFA-1 (leukocyte function-associated antigen), CD8 et CD3 agrégées à un pôle du lymphocyte $T$, qui constitue les vestiges de la synapse immunologique. Au pôle opposé, on observe une concentration de la protéine kinase $C \zeta(P K C \zeta)$ - un composant impliqué dans la polarité cellulaire. Cette répartition moléculaire est maintenue dans les cellules T en cours de mitose. Ces résultats suggèrent que la signalisation émanant de la synapse immunologique induit une mitose asymétrique qui aboutit à une partition inégale des molécules dans les 2 cellules filles.
En suivant la prolifération de ces cellules T grâce au composé fluorescent qui se dilue à chaque division cellulaire, Chang et al. ont observé que les cellules T qui ont fait une seule division, $48 \mathrm{~h}$ après leur transfert, peuvent être séparées en 2 souspopulations exprimant le co-récepteur CD8 à un fort (CD8 $\left.8^{\text {hi }}\right)$ ou un faible $\left(C D 8^{10}\right)$ niveau [7]. Les auteurs suggèrent que ces 2 sous-populations proviennent des cellules filles générées respectivement au pôle proximal et au pôle distal de la synapse immunologique initiale. Après purification de ces 2 populations, les auteurs ont pu établir que la population $C D 8^{\text {hi }}$ exprime un phénotype caractéristique de cellule T effectrice, tandis que la population $\mathrm{CD}^{\text {lo }}$ a plutôt un phénotype de cellule $T$ mémoire. L'injection de l'une ou l'autre de ces populations cellulaires dans une souris hôte immédiatement infectée par Listeria monocytogenes permet de contrôler l'infection. En revanche, si l'infection de
I'hôte survient 30 jours après le transfert des lymphocytes $T$, la population CD $8^{\text {hi }}$ est plus efficace que la population $\operatorname{CD} 8^{\text {lo }}$ pour contrôler cette infection tardive. Les auteurs suggèrent que les descendants d'un lymphocyte activé T CD8 héritent d'un matériel moléculaire disparate: la cellule fille recueillant les molécules concentrées au pôle proximal de la synapse immunologique (TCR, CD8 et LFA-1) se différencie en cellule $T$ effectrice, tandis que celle qui reçoit les molécules ségrégées au pôle

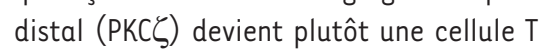
mémoire.

L'observation majeure rapportée par Chang et al. est donc la description de la division asymétrique des lymphocytes T. À ce stade, il n'y a pas encore de preuve directe que les 2 populations $\operatorname{CD} 8^{\text {hi }}$ et $C D 8^{\text {lo }}$ caractérisées $48 \mathrm{~h}$ après l'infection dérivent réellement de la même cellule $T$ qui subissait une mitose asymétrique. En effet, à ce jour, les techniques disponibles ne permettent

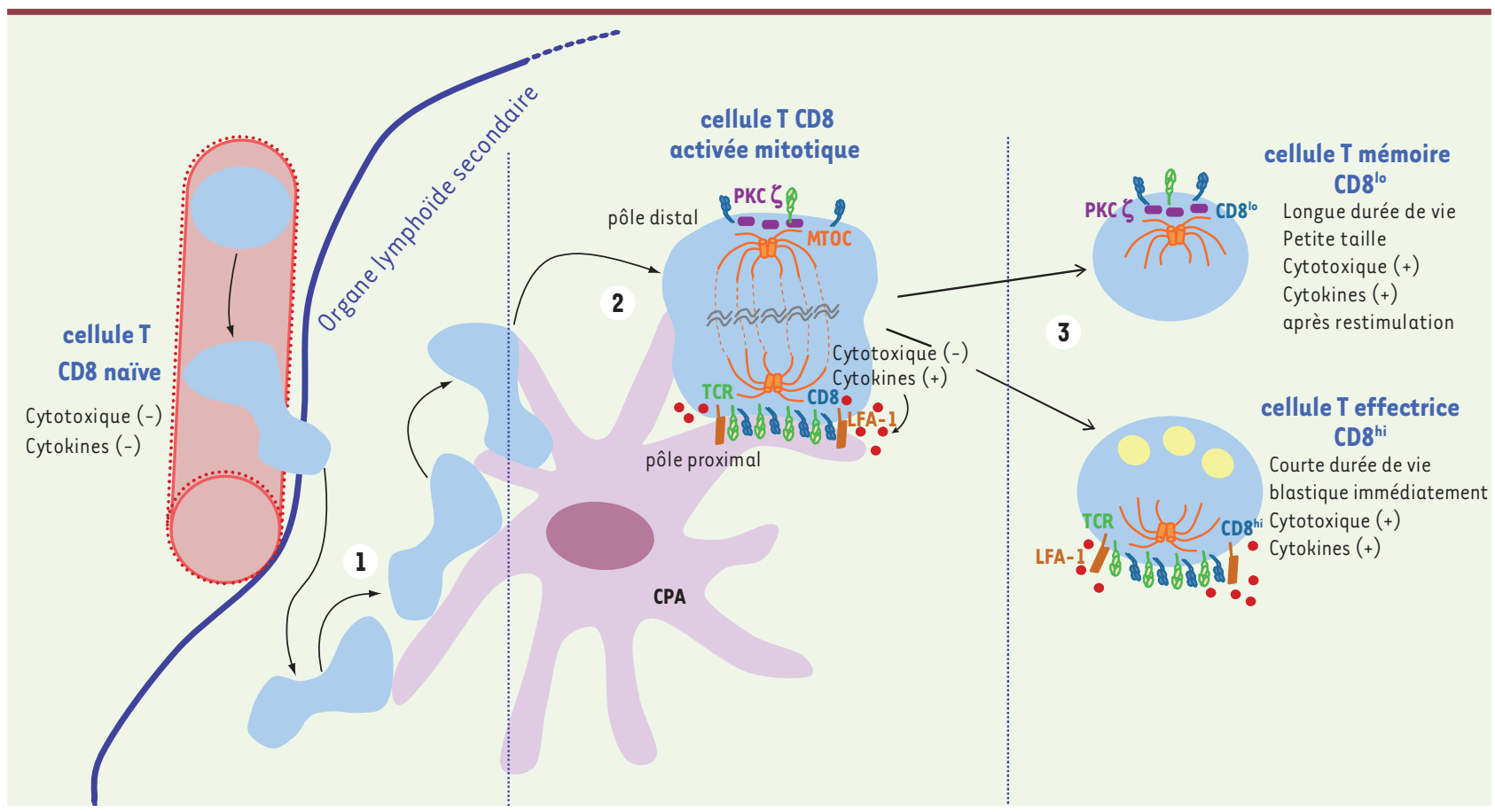

Figure 2. Cinétique d'activation et de différenciation des lymphocytes T CD8 mettant en jeu une division asymétrique. Les lymphocytes T migrent continuellement du sang vers les ganglions lymphatiques. Phase 1:0-6 h-Grande mobilité des lymphocytes T; interactions de courte durée avec la CPA. Phase $2: 6-32 \mathrm{~h}$ - Interaction stable (> $1 \mathrm{~h}$ ) du lymphocyte T avec la CPA. La formation de la synapse immunologique induit l'activation de la cellule T (expression de marqueurs d'activation, sécrétion de cytokines) et son entrée en mitose : ségrégation asymétrique des molécules d'activation aux 2 pôles de la cellule. Phase $3: 32-72 \mathrm{~h}$ - Le lymphocyte T redevient mobile. La division cellulaire engendre 2 cellules filles différentes : une cellule CD $8{ }^{\text {hi }}$ exprimant un phénotype caractéristique de cellule T effectrice (contenant des granules cytotoxiques, représentés en jaune), et une cellule $\mathrm{CD} 8^{10}$ ayant plutôt un phénotype de cellule T mémoire. 
pas d'isoler les cellules filles montrant à $32 \mathrm{~h}$ une répartition inégale de $\mathrm{PKC \zeta}$ et du TCR et de suivre le devenir de ces cellules individuelles dans le temps.

Les 2 populations de cellules TCD $8^{\text {hi }}$ et CD $8{ }^{\text {lo }}$ diffèrent par leur taille et leur granulosité, des modifications morphologiques observées lors de la différenciation des cellules T naïves en cellules T activées blastiques et qui s'accompagnent de l'augmentation de certains récepteurs à la surface. Dans le modèle utilisé par les auteurs, on ne peut exclure une réponse non synchronisée des cellules T transférées: les cellules T ayant rencontré l'Ag précocement seraient alors activées et blastiques; tandis que les cellules T ayant été stimulées à un temps plus tardif seraient en cours d'activation/ différenciation et n'auraient pas encore subi le changement métabolique et morphologique conduisant à la blastogenèse.
Selon cette hypothèse, la différence d'expression du co-récepteur CD8 reflèterait plutôt 2 stades distincts du processus de différenciation des lymphocytes T. Malgré cette restriction, les données de Chang et al. montrent clairement que ces 2 populations $\mathrm{CD} 8^{\text {hi }}$ et $\mathrm{CD} 8^{\text {lo }}$ génèrent in vivo respectivement, des cellules $T$ effectrices et mémoires.

La génération de divers types cellulaires via un processus de division asymétrique qui sépare inégalement les déterminants cellulaires est un mécanisme conservé dans l'évolution, depuis Caenorhabditis elegans et Drosophila melanogaster [8,9] jusqu'aux vertébrés. Elle pourrait, entre autres, être à l'origine de la grande plasticité des réponses des cellules du système immunitaire. $\diamond$ Asymmetric heritage in the daughter cells determines effector or memory cell fate in T lymphocytes

\section{NOUVELle}

\section{L'enzyme de la glycolyse GAPDH joue un double rôle dans la neurotransmission GABAergique et l'épilepsie humaine}

Jacques Laschet, René Pumain

\section{RÉFÉRENCES}

1. Bismuth G, Trautmann A. La synapse immunologique : des modèles aux réalités. Med Sci (Paris) 2006; $22: 721-6$.

2. Monks CR, Freiberg BA, Kupfer H, et al. Threedimensional segregation of supramolecular activation clusters in T cells. Nature 1998; 395 : 82-6.

3. Saito T, Yokosuka T. Immunological synapse and microclusters : the site for recognition and activation of T cells. Curr Opin Immunol 2006; 18 : 305-13.

4. Henrickson $S E$, von Andrian UH. Single-cell dynamics of T-cell priming. Curr Opin Immunol 2007 ; 19 : 249-58.

5. Kalia V, Sarkar S, Gourley TS, et al. Differentiation of memory B and T cells. Curr Opin Immunol 2006; 18: 255-64.

6. Sallusto F, Geginat J, Lanzavecchia A. Central memory and effector memory $T$ cell subsets : function, generation, and maintenance. Annu Rev Immunol $2004 ; 22: 745-63$.

7. Chang JT, Palanivel VR, Kinjyo I, et al. Asymmetric T lymphocyte division in the initiation of adaptive immune responses. Science 2007 ; 315 : 1687-91.

8. Betschinger J, Knoblich JA. Dare to be different: asymmetric cell division in Drosophila, C. elegans and vertebrates. Curr Biol 2004 ; 14 : R674-85.

9. Jagut M, Huyhn JR. Régulation des cellules souches de la lignée germinale : la niche s'agrandit chez la drosophile. Med Sci (Paris) 2007 ; 23 : 611-8.
Laboratoire de Neurobiologie

et Pharmacologie Moléculaire, Inserm U573, Centre Paul Broca,

2ter rue d'Alésia, F75014 Paris, France. jacques.laschet@broca.inserm.fr

des neurones hippocampiques ou corticaux dissociés de façon aiguë [4]. Les caractéristiques de ce mécanisme vont être décrites, ainsi que son altération dans les structures nerveuses à l'origine des crises d'épilepsie.

Un nouveau mécanisme: la modulation glycolyse-dépendante de l'inhibition

Le premier point essentiel était d'identifier la kinase endogène du GABA $A$ R. À notre grande surprise, la protéine ciblée après purification du récepteur et essais de phosphorylation ne faisait pas partie des kinases connues, mais correspondait à une enzyme clé de la glycolyse, la glycéraldéhyde-3-phosphate déshydrogénase (GAPDH) [5]. Un examen attentif de la
${ }^{1}$ Incapacitation (rundown), ce qui traduit une perte fonctionnelle au cours du temps indépendante de l'application de l'agoniste.
> De nombreux systèmes de neurotransmission sont modulés par des phosphorylations de récepteurs synaptiques dans le système nerveux central (SNC) des vertébrés. II a été en particulier établi que les récepteurs ionotropes au glutamate et au GABA intracellulaires de plusieurs des sous-unités qui les composent par des kinases impliqués dans la stabilité des récepteurs à la membrane plasmique et peuvent jouer un rôle essentiel dans la désensibilisation, l'agrégation, l'internalisation, le recyclage ou la dégradation de ces entités protéiques (pour revue, voir [1]). En ce qui concerne le récepteur de type $A$ au $G A B A\left(G A B A_{A} R\right)$, qui est composé de cinq sous-unités dont les plus fréquentes sont les $\alpha$, $\beta$, et $\gamma$, ces ases classiques phosphorylent essentie les sous-unités $\beta$ ou $\gamma$ du GAB Un mécanisme différent de phosphorylation a été décrit pour ce récepteur, endogène doit donc êtrétroitemer rylation prévient le rundown ou «incapacitation $»^{1}$ de la réponse $G A B A e r g i q u e$ 